\title{
Plausibilistic Preprocessing of Sparse Range Images
}

\author{
Björn Krebs, Bernd Korn and Friedrich M. Wahl \\ Institute for Robotics and Computer Control, \\ Technical University Braunschweig, \\ Hamburger Str. 267, D-38114 Braunschweig, F.R.G.
}

\begin{abstract}
Range image interpretation often suffers from contaminating noise and sparseness of the input data. Non-Gaussian errors occur if the physical conditions in the scene violate sensor restrictions. To deal with such drawbacks we present a new approach for range image preprocessing. To provide dense range information initial sparse data is augmented via appropriate interpolation. Furthermore, we propose a measure of plausibility which depends on the density of the initial data to judge the result of the interpolation.
\end{abstract}

Range image preprocessing - perspective distortions - non-Gaussian errors sparse range data - plausibility

\section{Introduction}

In many fields, e g. advanced robot applications, $3 \mathrm{~d}$ scene interpretation becomes more and more important. Range images are an appropriate interface between sensor systems and scene interpretation. Usually, the first step in scene analysis is to extract $3 \mathrm{~d}$ primitives representing surface or edge descriptions (e. g. planar patches [4], [5] or simple $3 \mathrm{~d}$ primitive shapes such as spheres or cylinders [2]). The use of fixed models restricts the scene interpretation to a specific class of detectable objects. To allow more flexible object models some authors propose burdensome direct matching of range data with freeform-surfaces (e. g. [3], [7]). In contrast to these well-known image analysis algorithms, our approach preprocesses range images without any a priori knowledge of the object surfaces. The preprocessing (subdivided into four layers) serves as an interface between the sensor dependent level and the more abstract level of $3 \mathrm{~d}$ scene interpretation: The first step is Range Image Acquisition; a subsequent Error Reduction has to rely on the used scanner (section 2). In the third layer the perspective distorted range information is transfered to functional data by Orthogonalization with simultaneous View Integration (section 3). To get dense range information an Augmentation in layer four interpolates the data (section 4). The interpolation has to obey the following criteria:

1. The original data must not be changed. 2 . The geometrical properties of the object surfaces, i. e. smoothness and curvature, must be preserved. 3 . The interpolated data need to be judged with respect to its reliability. Hence, the result of the interpolation has to be judged by a measure of plausibility. 


\section{Range Image Acquisition and Error Reduction}

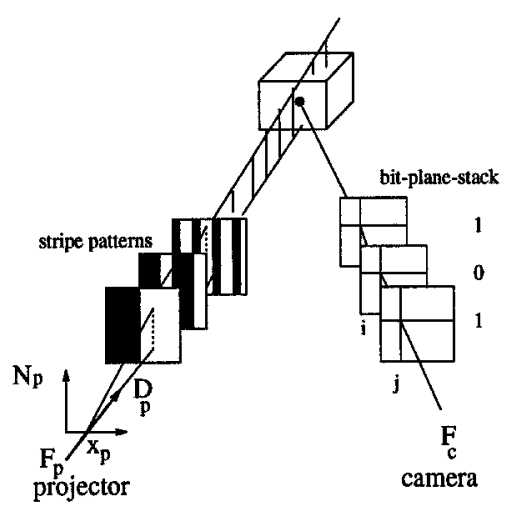

Fig. 1. Time-space encoding of the measurement area by means of $n$ stripe patterns.

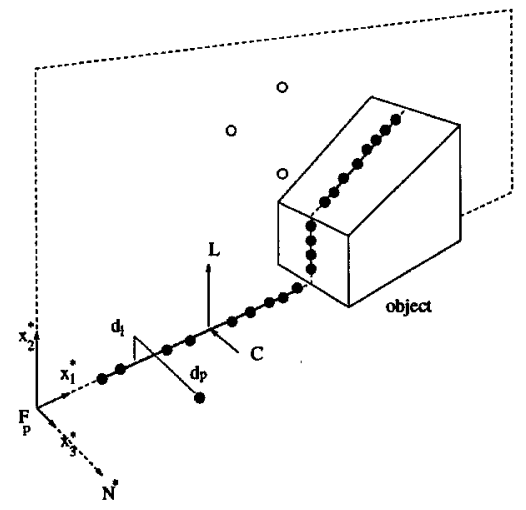

Fig. 2. All points which do not form a cut with an object surface are eliminated.

The used range sensor is based on the coded light approach which is a well-known fast and robust active triangulation technique (e. g. [9]). The system contains a camera, projectors and dedicated image processing hardware. A projector illuminates the scene with a sequence of $n$ stripe patterns, allowing a distinction of $2^{n}$ different illumination wedges and the extraction of the corresponding illumination planes (see figure 1 ). The $3 \mathrm{~d}$ coordinates of the observed scene points can be computed by triangulation, i. e. the intersection between the view line and the illumination plane.

In our experimental setup we use a depth-eye-in-hand configuration where the camera is mounted on a robot's hand while the projectors are installed rigidly around the work space of the robot. To supply different views, efficient recalibration and triangulation has been implemented in special image processing hardware $^{1}([8])$. However, raw sensor data is often sparse and contaminated with noise and non-Gaussian errors. The type of errors depends on the physics of the used range sensor. Lens distortions are eliminated in the triangulation step itself [8]. The non-random errors occur very often in exclusive parts of the image $^{2}$. An appropriate approach is to select only samples of data points which fulfill specific geometrical constraints. By sorting all point with respect to their distance to the plane origin a sample of points $\forall P \in S=\left\{P_{0}, \ldots, P_{n}\right\}$ with $\mathbf{n}$ elements in the illumination plane (defined by the normal vector $N^{*}$ and the plane origin $F_{p}$ ) can be judged if they define a cut with an object surface (see figure 2).

$$
n>\nu
$$

\footnotetext{
${ }^{1}$ DATACUBE MAX-VIDEO 10

2 especially near jump edges
} 

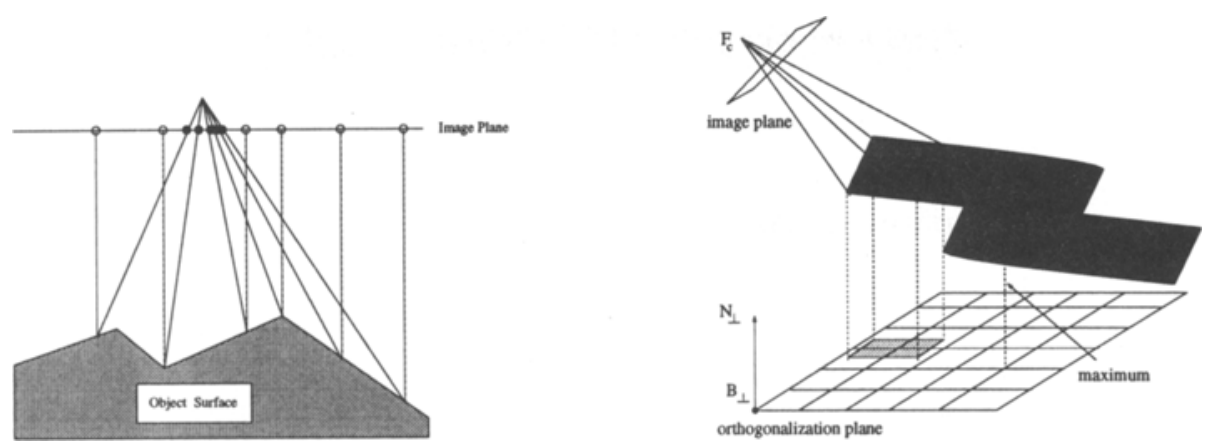

Fig. 3. Perspective and orthographic projections.

$$
\begin{aligned}
& d_{p}=\left|x_{3}^{*}\right|<\theta \\
& d_{l}=\mid\left(\left(x_{1}^{*} l_{1}+x_{2}^{*} l_{2}\right)-\left(c_{1} l_{1}+c_{2} l_{2}\right) \mid<\zeta\right.
\end{aligned}
$$

$L=\left(l_{1}, l_{2}\right)$ is the normal vector and $C=\left(c_{1}, c_{2}\right)$ the centroid of a straight line through the sample (coordinates with respect to the local coordinate frame of the illumination plane). The error reduction is controlled by the thresholds $\nu, \theta$ and $\zeta$.

\section{Perspective Distortions and Multiple View Integration}

Usually, range images suffer from perspective distortions. Generally, this is true for any type of range image because the data is generated via a perspective map (see left image of figure 3). Nevertheless, many applications assume long focal distances with almost parallel view-lines and therefore no perspective effects (e. g. [1]). But in real world ranging systems the operational distance, and therefore the focal distance as well, is short. To apply homogeneous filter operations ${ }^{3}$ without any further geometric distortions the perspective distorted range data has to be transferred to functional data by orthogonalization onto a orthogonalization plane. The orthogonalization restricts the observed surfaces to Monge surfaces which preserve their geometrical properties under orthogonalization ([6]). If a part of the object surface is non-Monge we select the maximum distance from the $3 \mathrm{~d}$ point to the orthogonalization plane $\mathrm{P}$ with the normal vector $N_{\perp}=\left(n_{1}, n_{2}, n_{3}\right)$ and the origin $B_{\perp}=\left(b_{1}, b_{2}, b_{3}\right)$ (see right image of figure 3 ).

$$
\pi_{\text {max }}\left(x_{1}, x_{2}, d_{\text {max }}\right): d_{\text {max }}=\max \left|\sum_{i=0}^{2} x_{i} n_{i}-\sum_{i=0}^{2} b_{i} n_{i}\right|
$$

The loss of information by orthogonalization of non-Monge surfaces can be handled by using more than one orthographic image: e. g. , orthographic maps in the three Cartesian coordinate system planes will supply all surface information.

$\overline{3}$ e. g. to extract gradients or surface normals 
But not all parts of a surface are visible. View integration solves this problem and can be easily achieved by projecting all data samples onto one orthogonalization plane.

\section{Augmentation of Sparse Data}

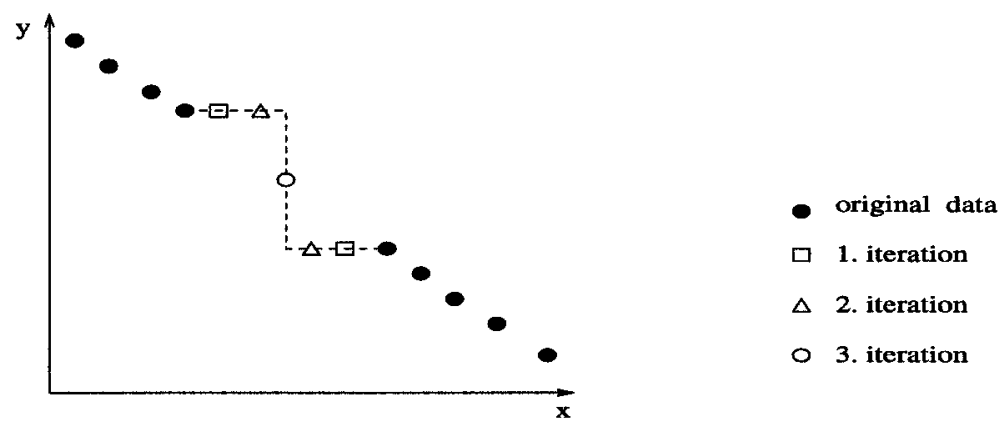

Fig. 4. Artefact produced by iterative sparse data averaging or median filtering.

The orthogonalization described above enables us to process a range image with similar homogeneous operations like greyscale images ${ }^{4}$. So, we can easily adapt well-known techniques from $2 \mathrm{~d}$ image processing like median or average filtering. Let $I=\{(x, y) \quad \mid \quad 0 \leq x \leq N, 0 \leq y \leq M\}$ be the set of pixels of an orthogonalized range image after error reduction. The range image is a function on an array of pixels $r_{0}:[0, \ldots, N] \times[0, \ldots, M] \mapsto \Re \quad \cup \quad\{\perp\}$ of valid range values $r_{0}(x, y) \in \Re$ or of unknown depth information $r_{0}(x, y)=\perp$. So, we can divide the range image into two disjoint sets of pixels:

$$
\begin{aligned}
& S_{0}=\left\{(x, y) \in I \quad \mid \quad r_{o}(x, y) \neq \perp\right\} \\
& \bar{S}_{0}=I-S_{0}
\end{aligned}
$$

A sparse data averaging filter can be realized with an $n \times n$ kernel $k_{n}(x, y)$ as follows:

$$
r_{i+1}(x, y)=\left\{\begin{array}{rll}
r_{i}(x, y) & : & (x, y) \in S_{i} \\
\sum_{Q} \frac{r_{i}(\xi, \eta)}{S_{i} \cap k_{n}(x, y) \mid} & : & (x, y) \notin S_{i}
\end{array}\right.
$$

and $Q=(\xi, \eta) \in\left(S_{i} \cap k_{n}(x, y)\right)$ and $S_{i+1}=\left\{(x, y) \in I \quad \mid \quad r_{i-1}(x, y) \neq \perp\right\}$.

The averaging operation tends to generate smooth edges. To preserve steep edges, we propose a sparse data median filter:

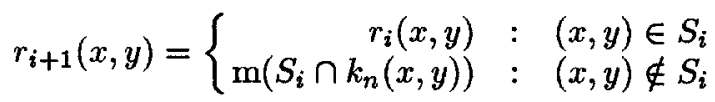

\footnotetext{
${ }^{4}$ Thus, common image processing hardware allows efficient computation
} 
with $\mathrm{m}(\mathrm{S})$ to be the median of the Set $\mathrm{S}$ of range values.

The update of $r_{i}(x, y)$ satisfies the first requirement of section 1 and the algorithm terminates already after a few iterations with $S_{i}=I$, depending on the cardinality of $\overline{S_{0}}$. However, in general the filter operation doesn't satisfy the second requirement (see figure 4).

Therefore we propose a kind of relaxation algorithm and modify the update in (7) to:

$$
r_{i+1}(x, y)=\left\{\begin{array}{rll}
r_{i}(x, y) & : & (x, y) \in S_{0} \\
\sum_{Q_{1}} \frac{r_{i}(\xi, \eta)}{n^{2}} & : & (x, y) \notin S_{0}
\end{array}\right.
$$

with $Q_{1}=(\xi, \eta) \in k_{n}(x, y)$.

The pixel $(x, y) \in \bar{S}_{0}$ must be initialized with an appropriate value $r(x, y) \in \Re$ and the algorithm stops when:

$\forall(x, y) \in I$

$$
\left|r_{i+1}(x, y)-r_{i}(x, y)\right| \leq \varepsilon, \varepsilon \in \Re^{+}
$$

is satisfied.

The third criterion we proposed in section 1 requires a measure of plausibility for the interpolated data. The plausibility of interpolated data $r(x, y)$ must increase with the number of measured data within the neighborhood of pixel $(x, y)$. Therefore we choose the initial plausibility

$$
p l_{0}=\left\{\begin{array}{lll}
1 & : & (x, y) \in S_{0} \\
0 & : & (x, y) \notin S_{0}
\end{array}\right.
$$

and the update

$$
p l_{i+1}=\left\{\begin{array}{rll}
p l_{i} & : & (x, y) \in S_{i} \\
\frac{1}{n^{2}-1} \sum_{Q_{1}} p l_{i}(\xi, \eta) & : & (x, y) \notin S_{i}
\end{array}\right.
$$

with $S_{0}, S_{i}, S_{i+1}$ as in (7)

and $Q_{1}$ as in (9).

\section{Experimental Results and Conclusions}

In our experiments the range images consist of $512 \times 512$ of 2 byte values. Only data near the borders of the illumination wedges provide proper values. Furthermore, highly erroneous data occur in some scene parts, e. g. due to occlusion. After the error reduction only $20 \%-30 \%$ of all pixels have depth values assigned. To supply complete surface information, six views of the whole scene are integrated; the orthogonalized image and the result of the preprocessing is shown in figure 5 .

In this paper we have proposed a surface and object model independent preprocessing of range images. Different images from several views can be integrated before scene analysis. With the introduced concept of geometrical constraints it is possible to select valid $3 \mathrm{~d}$ data. A subsequent augmentation provides dense range information judged by a plausibility measure. First solutions and results 

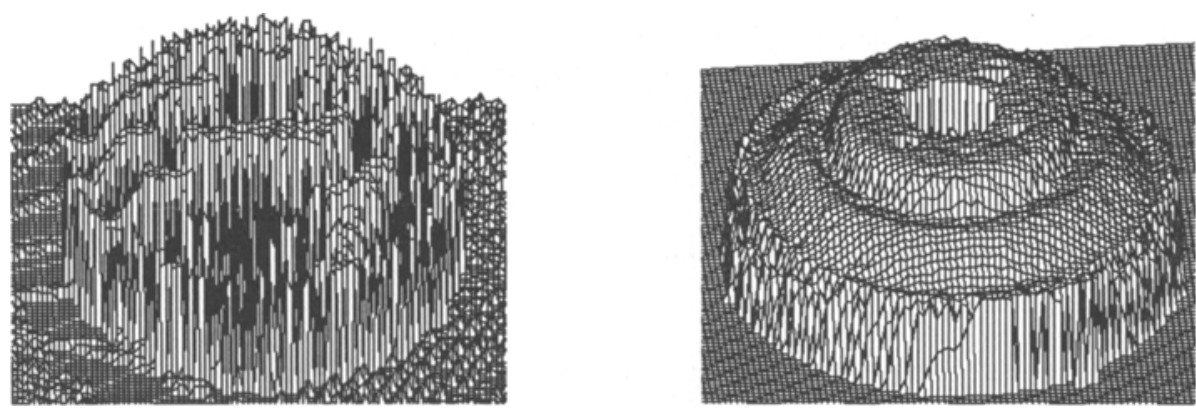

Fig. 5. The left image shows orthogonalized data of six integrated views. The right image shows the resulting $3 \mathrm{~d}$ information of the preprocessed range data with a plausibility greater than 0.1 . For a better visualization $r_{0}(x, y)=\perp$ was replaced by $r_{0}(x, y)=-1$.

have been outlined and the proposed algorithm has been validated with real world range images. In future, the preprocessing will be fully integrated in our scene interpretation system to support more complex surface models. Furthermore, the range image acquisition and preprocessing will support sensor guided grasping and assembly currently investigated and developed at our institute.

\section{References}

1. P. J. Besl and R. C. Jain. Segmentation through variable-order surface fitting. IEEE Transactions on Pattern Analysis and Machine Intelligence, 10(2):167-192, 1988.

2. R. Bolles and M. A. Fischer. A ransac-based approach to model fitting and its application finding cylinders in range data. In 7 th Int. Conf. on Art. Int. , Vancouver, pages $637-643,1981$.

3. K. Higuchi, M. Hebert, and K. Ikeuchi. Bulding 3-d models from unregisterd range images. In Proc. IEEE Conference of Robotics and Automation, San Diego, CA, pages $2248-2253,1994$.

4. J. M. Jolion, P. Meer, and S. Bataouche. Robust clustering with applications in computer vision. Pattern recognition, 13(8):791-802, 1991.

5. R. Krishnapuram and C. P. Freg. Fitting an unknown number of lines and planes to image data combatible cluster merging. Pattern Recognition, 25(4):385-400, 1992.

6. M. A. Penna and S. Chen. Image Understanding in Unstructured Enviroment, volume 2 of Series in Automation, chapter Sperical Analysis in Computer Vision and Image Understanding, pages 126-177. World Scientific, 1994.

7. D. A. Simon, M. Hebert, and T. Kanade. Real-time 3-d pose estimation using a high-speed range sensor. In Proc. IEEE Int. Conf. on Robotics and Automation, San Diego, CA, volume 3, pages 2235-2240, 1994.

8. T. Stahs and F. M. Wahl. Fast and Versatile Range Data Acquisition in a Robot Work Cell. In Proc. IEEE Int. Conf. on on Intelligent Robots and Systems (IROS) 1992, Raleigh, North Carolina, 1992.

9. F. M. Wahl. A Coded Light Approach for 3D Vision. In IBM Research Report, RZ $1452,1984$. 